\title{
Detection and Characterization of Transiting Systems with Smaller Exoplanets
}

\author{
Teruyuki Hirano ${ }^{1}$, Norio Narita ${ }^{2}$, Akihiko Fukui ${ }^{2}$, \\ Roberto Sanchis-Ojeda ${ }^{3}$, Joshua N. Winn ${ }^{3}$, Yasushi Suto ${ }^{1}$, \\ and Atsushi Taruya ${ }^{1}$ \\ ${ }^{1}$ Department of Physics, The University of Tokyo, Tokyo 113-0033, Japan \\ email: hirano@utap.phys.s.u-tokyo.ac.jp \\ ${ }^{2}$ National Astronomical Observatory of Japan, 2-21-1 Osawa, Mitaka, Tokyo, 181-8588, Japan \\ ${ }^{3}$ Department of Physics, and Kavli Institute for Astrophysics and Space Research, \\ Massachusetts Institute of Technology, Cambridge, MA 02139, USA
}

\begin{abstract}
We present our effort to detect and characterize transiting systems with small exoplanets. First we present our on-going project to detect transiting exoplanets around late-type stars. As many authors point out, late-type stars can host potentially habitable rocky planets, whose transits are detectable with 2-meter class telescopes. We aim at detecting (small-sized) transiting exoplanets around M-type stars and are conducting a survey using the Okayama $1.88 \mathrm{~m}$ telescope. We introduce our campaign at Okayama. Next we discuss the characterization of small-sized exoplanets. We focus on the measurement of the spin-orbit angle, the angle between the stellar spin axis and planetary orbital axis. The spin-orbit relations are of great importance in discussing planetary formations, evolutions, and migrations. To this point, the Rossiter-McLaughlin (RM) effect, an apparent radial velocity anomaly during a planetary transit, has been mainly investigated to measure the projected spin-orbit angles. However, as the size of the transiting planet becomes smaller, the detection of the RM effect becomes challenging because of the small RM signal. We have newly developed a technique to investigate spin-orbit relations for smaller planets by combining Kepler's ultra-precise photometry and spectroscopic measurements. We show that, contrary to planetary systems with close-in giant planets, most of the systems with small-sized planets (including Earth-sized ones) have smaller spin-orbit angles, which implies a different evolutional history of the planetary systems. We also discuss future prospects on the detection and characterization of smaller transiting exoplanets.
\end{abstract}

Keywords. techniques: photometric, techniques: spectroscopic, stars: rotation, stars: spots

\section{Introduction}

Recent dedicated missions with both space and ground-based telescopes have enabled detections of many smaller exoplanets (candidates) including Earth-sized ones. Those missions have revealed that such smaller planets are more abundant in number compared to jovian planets (e.g., Borucki et al. 2010), and have a diversity in terms of their orbital elements and internal structures.

We here describe the detection and characterization of smaller exoplanets using groundbased telescopes. For the detection of smaller exoplanets, we introduce our ongoing campaign of transit observations using the Okayama $188 \mathrm{~cm}$ telescope. Based on the reanalysis of the archived data of the successful transit survey (i.e., the superWASP survey, Pollacco et al. 2006), we attempt to find small-sized planets (down to Earth-sized ones) around cool dwarfs (M-type stars in particular). As for the characterization of small planets, we focus on measurements of the spin-orbit angles, which is tightly related to the evolution history of exoplanetary systems. We point out an important limitation of 
Table 1. Specification of Okayama/ISLE

\begin{tabular}{c|c}
\hline Array & HAWAII $1 \mathrm{~K} \times 1 \mathrm{~K} \mathrm{HgCdTe}$ \\
FOV & $4^{\prime} .3 \times 4^{\prime} .3$ \\
Scale & $0.245 " \mathrm{pix}^{-1}$ \\
Filters & $J, H, K_{\mathrm{s}}, H K$ \\
\hline
\end{tabular}

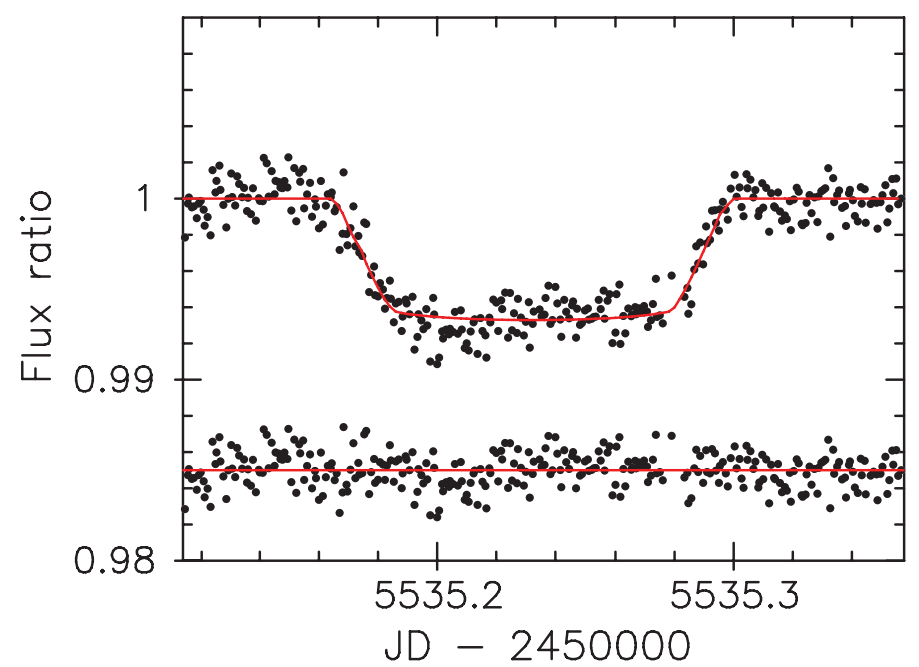

Figure 1. A transit lightcurve taken by OAO/ISLE. The residual of the observed flux from the best-fit model is shown at the bottom.

observing the Rossiter-McLaughlin effect to measure spin-orbit angles, and suggest an alternative method for that purpose using the precise photometry taken by the Kepler space telescope.

\section{Detection of Smaller Exoplanets}

For the detection of smaller (Earth-sized) exoplanets, cool stars are widely believed to be good targets. Specifically, we focus on transiting planets around M dwarfs for the following reasons:

(a) M dwarfs are small (in terms of radius) and thus transits are likely deeper,

(b) M dwarfs are less massive, which facilitates radial velocity measurements,

(c) M dwarfs are the most numerous in the proximity of our solar system,

(d) M dwarfs are cool and hence the habitable zone is relatively closer to the stars. One issue in attempting to observe $M$ dwarfs is their faintness in the optical wavelength. In order to achieve a higher signal-to-noise ratio (SNR), the simplest way to go is to observe the $\mathrm{M}$ dwarfs in the near infra-red (NIR) region. We here present our effort to discover smaller planets around M dwarfs using the Okayama $188 \mathrm{~cm}$ telescope.

In order to achieve a precise photometry specified for transit observations, we exploit the NIR instrument "ISLE" mounted on the $188 \mathrm{~cm}$ telescope at Okayama Astrophysical Observatory (OAO). Table 1 summarizes the specification of OAO/ISLE. For ISLE observations, we can employ either the imaging and spectroscopy modes, and achieve the spectral resolution of $R=300-4,000$ for the spectroscopy mode. To maximize the photometric precision and avoid systematics, we make use of the auto-guiding system in taking transit lightcurves and OAO/ISLE has demonstrated that approximately $1 \mathrm{mmag}$ precision could be steadily achieved for stars with $J<10$ mag. For instance, in Figure 1 , 
Table 2. Comparison of ground-based photometric precisions in NIR

\begin{tabular}{ccccc}
\hline Telescope (diameter $[\mathrm{m}])$ & Instrument & Object & Filter & Photometric precision (60-s binned) \\
\hline TCST $(1.52)$ & CAIN-II & GJ 436 & $H$ & $0.56 \mathrm{mmag}$ \\
CFHT(3.6) & WIRCam & WASP-12 & $J$ & $0.75 \mathrm{mmag}$ \\
OAO (1.88) & ISLE & HAT-P-13 & $J$ & $1.1 \mathrm{mmag}$ \\
VLT $(8.2)$ & ISAAC & WASP-4 & $K_{s}$ & $1.1 \mathrm{mmag}$ \\
WHT $(4.2)$ & LIRIS & TrES-3 & $K$ & $2.0 \mathrm{mmag}$ \\
\hline
\end{tabular}

we show a transit lightcurve observed by ISLE for HAT-P-13. The exposure time for this case was fixed at $60 \mathrm{sec}$, and $1.1 \mathrm{mmag}$ precision could be achieved from the analysis of this transit lightcurve. A photometric precision of $\sim 1$ mmag roughly corresponds to detections of super-Earths around M dwarfs. Comparison of ISLE's photometric precisions with other NIR instruments is summarized in Table 2.

For the 2012B semester, we are given 23 nights at OAO. In order to maximize the telescope's productivity, we adopt the following strategy; we reanalyze the archived data of selected surveys (e.g., the superWASP survey), and pick up candidates for transiting planets by implementing our own periodic analysis of the archived lightcurves specifically for cool dwarfs. Then, the potential candidates are subjected to the transit observations at $\mathrm{OAO}$ based on the new transit ephemeris for each target. If any of the targets shows a transit-like event, we move on to follow-up RV measurements with High Dispersion Spectrograph (HDS), followed by the direct imaging by High-Contrast Instrument with Adaptive Optics (HiCIAO) both installed on the Subaru $8.2 \mathrm{~m}$ telescope.

To this point, we have attempted to observe more than 20 cool dwarfs, which were picked up by our reanalysis of the superWASP archived data. While we have ruled out most of those stars as false alarms for transit candidates, some stars show flux variations and remain in our candidate list. Due to the bad weather or the small relative radii of potential candidates, however, we have not obtained a robust detection of planetary transits. Those systems will be subjected to further attempts of transit observations and RV follow-up measurements are also scheduled with Subaru/HDS. We hope to present the final results of our campaign somewhere in due course.

\section{Characterization of Smaller Exoplanets}

The second topic here is the characterization of small planets. Specifically, as a characterization of exoplanetary systems, we have focused on the investigation of "spin-orbit angles". It is widely believed that giant planets are created beyond the "snow line", which is located at a few AU away from the central star. But since the discovery of close-in giant planets (e.g., 51 Pegasi b), planetary migrations have been discussed to account for their close-in orbits (e.g., Lin et al. 1996).

The relation between the stellar spin axis and planetary orbital axis is of great importance in the context of planetary migrations in exoplanetary systems (Queloz et al. 2000, Winn et al. 2005, Ohta et al. 2005). This is because different migration processes predict different outcomes for the spin-orbit relation, and hence measurements of spin-orbit angles become an important observational clue to confirm or refute possible hypothesis on planetary migrations. Migrations due to the gravitational interaction between planets and proto-planetary disk are quiescent, and conserve the initial direction of planetary orbital planes (e.g., Lin et al. 1996). On the other hand, migrations including a scattering 

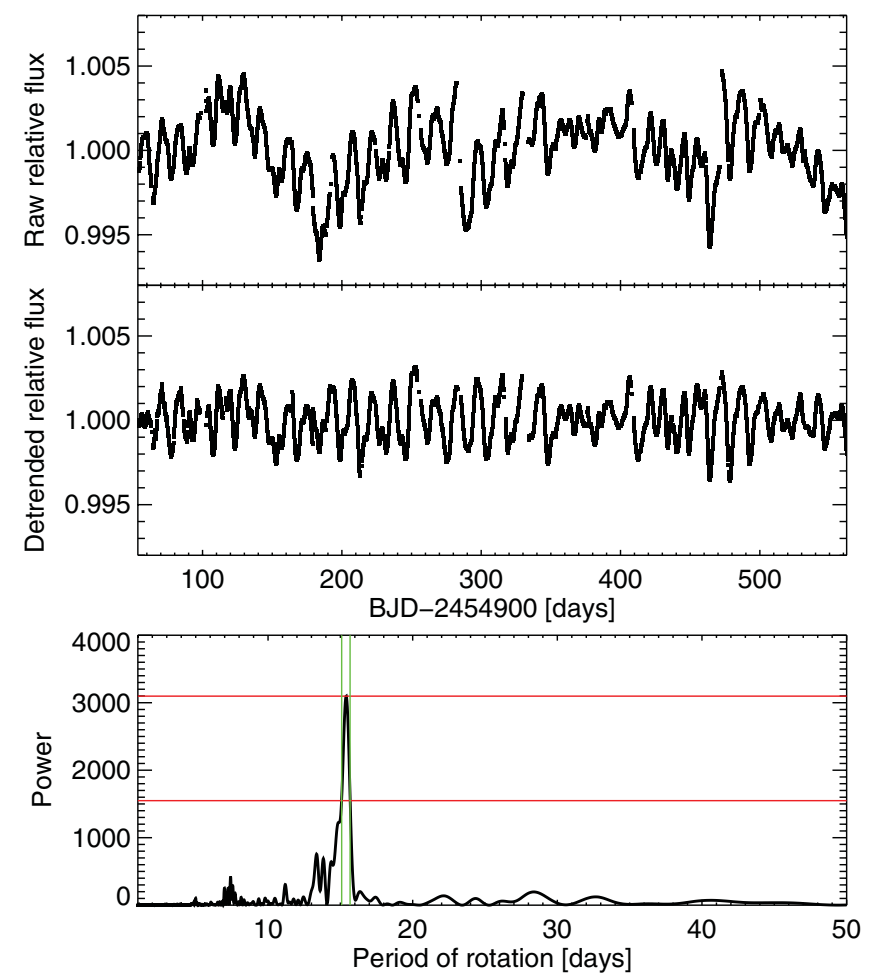

Figure 2. Top. - Quarter by quarter mean normalized raw out-of-transit flux of KOI-261 (PDC SAP_FLUX). Includes quarters 0 through 6 , except quarter 5 where this target was not observed. Central.- Detrended flux of the star. Bottom. - Lomb-Scargle periodogram of the detrended flux. The solid vertical lines represent the points where the power is half of the maximum power. The final value is taken to be the mean of these points, and the error to be half of their difference (Hirano et al. 2012).

process between multiple planets may dramatically change the direction of the planetary angular momentum and sometimes lead to spin-orbit "misalignments" (e.g., Rasio \& Ford 1996, Nagasawa et al. 2008). If a planetary orbit becomes highly eccentric due to planet-planet scattering, tidal interaction between the planet and its host star begins to operate around the periastron, and consequently shrink the planetary semi-major axis, thus resulting in close-in giant planets.

So far, measurements of the Rossiter-McLaughlin (RM) effect is the only major method to estimate the spin-orbit angles. The RM effect is an distortion in spectral lines when a portion of the rotating stellar disk is occulted by a transiting planet. This effect was measured for the first time in the system of CoRoT-2b (Bouchy et al. 2008). Through the RM effect, we can measure the sky-projected angle between the stellar spin axis and planetary orbital axis, usually denoted by $\lambda$. This technique to measure spin-orbit angles has been very successful and more than fifty systems have been investigated to date. However, we should keep in mind that there is an important limitation of the RM measurements; when the size of the planet is smaller (e.g., Earth-sized ones), the detection of the RM effect becomes challenging. This is because the signal of the anomalous radial velocity due to the RM effect is basically proportional to the transit depth (instantaneous loss of light due to transit), and small RM signals for the case of Earth-sized planets are beyond the velocity precision of our current technique. 


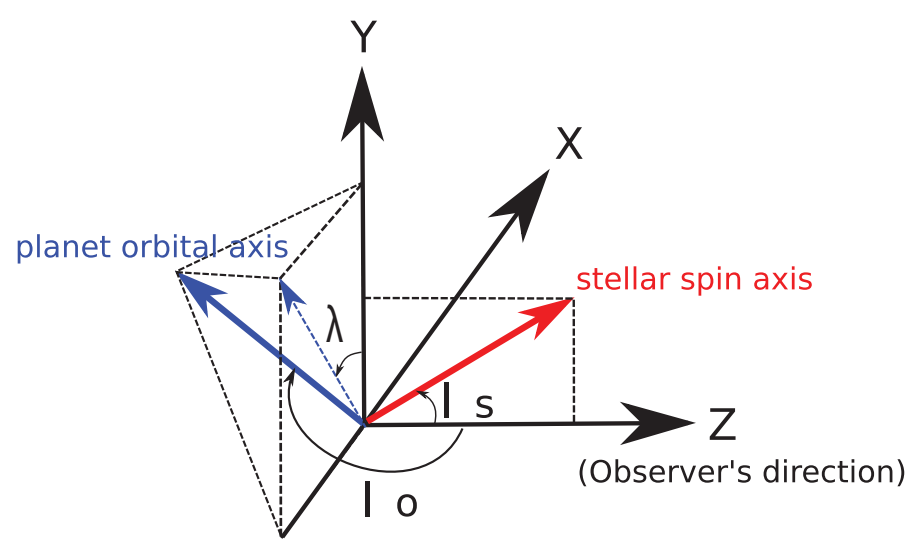

Figure 3. Schematic figure for the spin and orbital axes. In this figure, the $x-y$ plane indicates the sky plane and the $z$ axis points toward us. The planet's orbital axis is indicated by the blue arrow and its projection onto the sky plane is shown by the dashed arrow. The red arrow is the stellar spin axis, which is located in the $y-z$ plane (Hirano et al. 2012).

In order to make it possible to measure spin-orbit angles for smaller exoplanets, we focus on the Kepler photometry. Thanks to the long-term monitoring with a high precision, we can see periodic flux variations for some active stars mainly caused by rotation of starspots on the stellar surface. In this case, we can infer the rotational period of the star by implementing a periodicity analysis. In Figure 2, we plot a sample lightcurve for KOI-261 and its periodogram at the bottom. The detrended lightcurve show a flux variation of $\sim 0.5 \%$, which is most likely caused by starspots. The single strong peak in the periodogram around $\sim 15$ days is interpreted as the rotational period of the star.

Usually, from a high dispersion spectroscopy, we can estimate the projected rotational velocity $V \sin I_{s}$ of the star by measuring the width of spectral lines. Also, a measurement of the stellar effective temperature and surface gravity (and metallicity) enables us to estimate the stellar radius given an isochrone model. All of these combined, we can measure the stellar inclination $I_{s}$, which is the angle between the line-of-sight and stellar spin axis by the following equation:

$$
I_{s}=\arcsin \left\{\frac{P_{s} V \sin I_{s}}{2 \pi R_{s}}\right\},
$$

where $P_{s}$ is the rotational period of the star estimated from the periodicity analysis of Kepler's lightcurve.

There is a remarkable advantage in obtaining the stellar inclination for a star hosting a transiting planet. Namely, we can put a constraint on the spin-orbit angles; since the orbital inclination of a transiting exoplanet (denoted by $I_{o}$ in Figure 3 ) is close to $90^{\circ}$, a small value of $I_{s}$ implies a spin-orbit misalignment. Although the condition $I_{s} \sim 90^{\circ}$ does not necessarily indicates a spin-orbit alignment (e.g., $\lambda \neq 0^{\circ}$ in Figure 3), the observed distribution of stellar inclinations provides some insight into the dependence of the spinorbit angles on the planet size, host star's effective temperature, and so on. This method to infer the spin-orbit relation can be applied regardless of the size of the planet, enabling us to discuss spin-orbit alignment/misalignment for smaller exoplanets.

Motivated by the above described advantage to measure stellar inclinations, we spectroscopically observed several systems, which were labeled as Kepler Objects of Interest (KOI). All the systems that we observed showed some flux variations likely caused by 


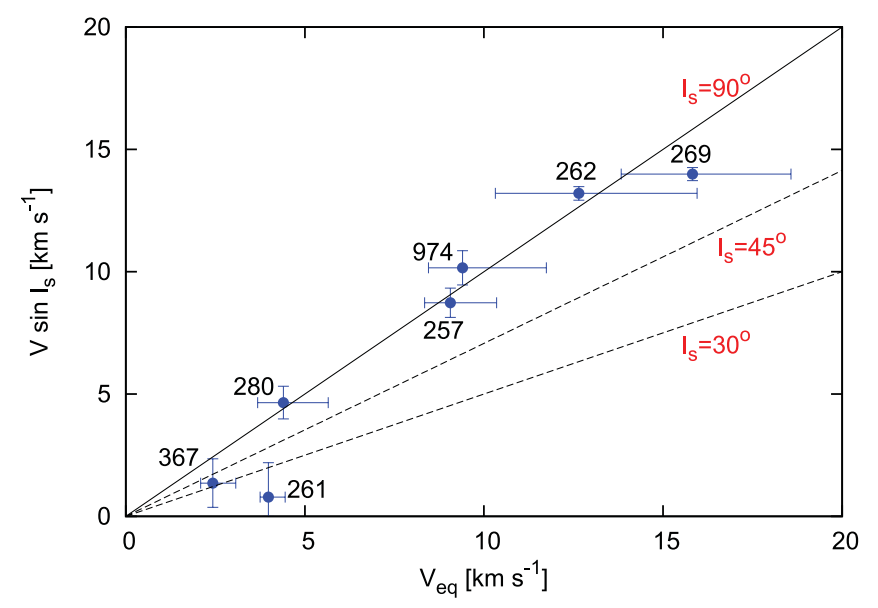

Figure 4. The estimated $V_{\text {eq }}$ and $V \sin I_{s}$. The solid line indicates the case that our line-of-sight is vertical to the stellar spin axis. We also plot the two inclined cases in which $I_{s}=45^{\circ}$ and $30^{\circ}$ (Hirano et al. 2012).

starspots. Prior to the observation, we analyzed the public lightcurves delivered by the Kepler team, and extracted the host stars' rotational periods $P_{s}$. The spectroscopic observation was performed with Subaru/HDS and we estimated the atmospheric parameters (e.g., $T_{\text {eff }}, \log g,[\mathrm{Fe} / \mathrm{H}]$ ) based on Takeda et al. (2002), and the projected rotational velocity $V \sin I_{s}$ by measuring the line widths. The atmospheric parameters were then converted into the mass, age, and radius of the host star based on the $\mathrm{Y}^{2}$ isochrone model (Yi et al. 2001).

Figure 4 plots thus estimated $V \sin I_{s}$ as a function of the stellar rotational velocity at the equator $V_{\mathrm{eq}} \equiv 2 \pi R_{s} / P_{s}$, which was estimated by the combination of the Kepler photometry and Subaru spectroscopy. The solid line in the figure denotes $V \sin I_{s}=V_{\text {eq }}$, which means the stellar inclination is nearly $90^{\circ}$. As the projected rotational velocity $V \sin I_{s}$ deviates from that solid line, the stellar inclination becomes smaller, suggesting a possible spin-orbit misalignment. Among the seven systems that we observed with Subaru/HDS, one system, KOI-261 shows a significant deviation from $I_{s}=90^{\circ}$, and may possibly have a spin-orbit misalignment. Systems with possible spin-orbit misalignments like KOI-261 are interesting targets for further investigations such as the measurement of the RM effect. We note that all the systems in Figure 4 have Neptune-sized or Earth-sized planet candidates.

\section{References}

Borucki, W. J., et al. 2010, Science, 327, 977

Bouchy, F., et al. 2008, A\&A A, 482, L25

Hirano, T., Sanchis-Ojeda, R., Takeda, Y., Narita, N., Winn, J. N., Taruya, A., \& Suto, Y. 2012, ApJ, 756, 66

Lin, D. N. C., Bodenheimer, P., \& Richardson, D. C. 1996, Nature, 380, 606

Nagasawa, M., Ida, S., \& Bessho, T. 2008, ApJ, 678, 498

Ohta, Y., Taruya, A., \& Suto, Y. 2005, ApJ, 622, 1118

Pollacco, D. L., et al. 2006, PASP, 118, 1407

Queloz, D., Eggenberger, A., Mayor, M., Perrier, C., Beuzit, J. L., Naef, D., Sivan, J. P., \& Udry, S. 2000, A\&A, 359, L13 
Rasio, F. A. \& Ford, E. B. 1996, Science, 274, 954

Takeda, Y., Ohkubo, M., \& Sadakane, K. 2002, PASJ, 54, 451

Winn, J. N., et al. 2005, ApJ, 631, 1215

Yi, S., Demarque, P., Kim, Y.-C., Lee, Y.-W., Ree, C. H., Lejeune, T., \& Barnes, S. 2001, ApJS, 136,417 